\title{
The rediscovery of nephropathology with artificial intelligence
}

\author{
Luís Rodrigues $^{1,2}$, Pinaki Sarder ${ }^{3}$, Kuang-Yu Jen ${ }^{4}$, Brandon Ginley ${ }^{3}$, Jorge Pratas ${ }^{1}$, Vitor Sousa ${ }^{2,5}$, Arnaldo Figueiredo ${ }^{2,6}$, Rui Alves ${ }^{1,2}$ \\ ${ }^{1}$ Nephrology Department, Centro Hospitalar e Universitário de Coimbra, Portugal \\ 2 Faculty of Medicine, University of Coimbra, Portugal \\ 3 University at Buffalo - The State University of New York \\ ${ }^{4}$ Department of Pathology and Laboratory Medicine, University of California, Davis School of Medicine \\ ${ }^{5}$ Anatomical Pathology Department, Centro Hospitalar e Universitário de Coimbra, Portugal \\ ${ }^{6}$ Urology and Renal Transplantation Department, Centro Hospitalar e Universitário de Coimbra, Portugal
}

\section{INTRODUCTION}

A kidney biopsy is a beloved tool for most nephrologists. It is as old as nephrology itself, and for the last fifty years, it has helped us to understand the pathophysiology of kidney disease and offer the best care to our patients. During this period, several technological advances have improved the excellence of this instrument: the percutaneous needle approach, ultra-sound guiding, novel immunohistochemistry (IHC) markers, immunofluorescence, and electron microscopy.

We are entering an exciting time of using artificial intelligence (AI) based and deep machine learning (ML) tools for delivering precision medicine. In pathology, high-resolution whole slide imaging (WSI) has enabled the development of digital pathology and computational image analysis algorithms to assist with diagnosis and classification. As we try to deepen our morphological analysis, we progressively develop more complex classification systems. To maintain optimal inter-observer reproducibility when applying these tools, the observer's need for learning and training increases in parallel. Digital pathology provides the ideal platform for investigating, teaching, and training new classifications. ML applied to our WSI will support reproducibility and reduce time-consuming tasks for the pathologist. These algorithms can extract complex morphometric information and correctly interpret data to study disease mechanisms, severity, predict outcomes, and eventually expose unique therapeutic targets.

The advances in oncopathology lead the way: skin, breast and thyroid cancer are examples where artificial intelligence (AI) applications are being developed. ${ }^{1-3}$ The adoption of $\mathrm{ML}$ tools in medicine is still at its launch, but the first medical device using Al, capable of diagnosing diabetic retinopathy without human intervention, has already been approved by regulatory authorities. ${ }^{4}$ Nephropathology will surely follow, and Al's potential for improving our patient care is remarkable.

\section{DIGITAL PATHOLOGY}

Digital pathology refers to the process of digitizing pathology glass slides using WSI scanners and encompasses the analysis of those images in a virtual environment, opened for the potential of computational resources. Over the past twenty years, we have witnessed the evolution of WSI scanners to the point where we can now scan a slide in less than 5 minutes. Digital pathology has already proven to have a diagnostic performance on a par with conventional microscopy-based approaches. The most crucial step for recognizing these technologies was taken in 2017 when the US Food and Drug Administration announced the approval of WSI for primary diagnosis in surgical pathology. ${ }^{5}$

The advantages of exploiting digital pathology range from everyday clinical practice to education and advanced translational research. The COVID19 pandemic has recently highlighted the remote exercise of medicine. With WSI, we can easily access our biopsies and even have them analyzed by experts from other institutions through teleconsultation networks. The establishment of digital pathology repositories is ongoing and has given rise to the concept of a digital biobank. These online digital slide collections are sources of big data that are already being used in nephrology by different research groups to study the pathogenesis of kidney disease. ${ }^{6}$

Like all new instruments developed in medicine, digital pathology must address several pending issues and limitations. Quality control for WSI, ranging from tissue processing to image scanning and analysis, must be standardized. Proper image labeling, multi-center validation and complying with patient data protection regulation will also have to be considered. The costs of institutional implementation of digital pathology are substantial. The minimum hardware includes a WSI scanner and large data capacity server storage with capable network bandwidth. Despite the initial investment, an increase in efficiency, operational utility, and savings in future pathology assets are expected. ${ }^{7}$

Perhaps the most prodigious element of digital pathology is enabling the computational analysis of our slides. We have evolved from the pioneer and promising pixel-based planimetry solutions to quantify fibrosis or the number of peritubular capillaries with IHC. The brilliant ideas of Alan Turing, considered to be the father of Al and notable cryptanalyst during the Second World War, are becoming real with the design of complex pattern recognition and self-learning algorithms in software engineering.

\section{ARTIFICIAL INTELLIGENCE IN NEPHROPATHOLOGY}

$\mathrm{Al}$ is rapidly proving to be essential for problem-solving in daily clinical practice. $\mathrm{ML}$ is a subfield of $\mathrm{Al}$, where algorithms can learn and improve from experience and are exceptionally useful in extracting 
value from big data. The development of ML tools will help clinicians achieve more efficient and precise decisions in various medical fields. In WSI analysis, the most popular and advanced ML algorithms are convolutional neural networks (CNN). A CNN is a deep learning algorithm used in object recognition that supports automated machine vision. These networks are essentially a group of artificial neurons that may undergo training to classify a subject image. Using a preset of image filters (such as edges, shapes, textures, or color), the CNN will detect patterns to form hidden convolutional layers and finally calculate a spatial likelihood heatmap for the desired output. The swift universal implementation of CNN in clinical practice requires that their WSI analysis is achieved through tissue samples processed by standard stains used in optical microscopy. However, multi-compartment segmentation training can include sophisticated methods, for instance, fluorescence-based multiplex IHC staining as ground-truth for training the computer. (Figure 1)

So far, breakthroughs in ML applications for WSI have been devoted to cancer pathology and have resulted in various tools proposed to enhance diagnostic precision and identify novel biomarkers. An excellent illustration was the development of an accurate ML classification model and decision support system using only reported diagnoses as labels for training. ${ }^{3}$ Its concept is to run this predictive model on each new scanned slide, and the algorithm will sort cases and slides within each patient based on the predicted tumor probability. The results showed that with the model's recommendations (through an interface that would flag positive slides), pathologists would be able to exclude $65-75 \%$ of slides from their workload while retaining $100 \%$ sensitivity.

In nephropathology, the first endeavors are occurring. Computational segmentation and classification of diabetic glomerulosclerosis have been achieved with comparable accuracy to human visual classification methods. ${ }^{8}$ Neural network segmentation of interstitial fibrosis, tubular atrophy, and glomerulosclerosis is also promising. ${ }^{9}$ Research groups are starting to address different areas of interest, where the introduction of these technologies would be most welcome in nephrology. Transplant pathology is a good example. CNN can prove extremely helpful in the study and prognostication of acute and chronic cellular and humoral rejection. The deceased donor kidney procurement biopsy is another significant problem to address. Current methods used for their classification discard approximately $50 \%$ of kidneys from marginal donors, suggesting that some could have been used with a more precise assessment. ${ }^{10,11}$ The computational fusion of large-scale histopathological information with donor clinical variables could improve our allograft prognosis predictions, increase organ usage, and optimize allocation.

Reproducibility is an everyday obstacle in the evolution of nephropathology. In addition to potentially unveiling kidney disease etiology, a kidney biopsy also allows the estimation of the degree of irreparable damage or chronicity in glomeruli, arteries, and the tubulointerstitial compartment. This information is often the most valuable since it is considered the best prognosis marker, irrespective of the etiology. ${ }^{12}$ In our daily practice, we try to group all these data into different classifications or scores, such as in IgA nephropathy, lupus nephritis (LN) or the Banff Classification of renal allograft pathology. For instance, in LN, the latest revised International Society of Nephrology/Renal Pathology Society classification includes class and activity and chronicity indexes that will provide diagnosis, prognosis, and assist with treatment options. ${ }^{13}$ These scoring methods offer good overall reproducibility when performed by experienced pathologists, but their consistency diminishes in less expert hands. ML tools promise the desired consistent precision that would undoubtedly be welcomed by all clinicians.

\section{Figure 1}

(A) Whole slide panoptic segmentation of interstitium (green), glomeruli (yellow), globally sclerotic glomeruli (red), tubules (blue), and large vessels (black) in a transplant renal biopsy. (B) Zoomed region of (A). (C) Fluorescence based multiplex IHC staining of six compartments: Type III Collagen, Leukocytes, Endothelial cells, Distal \& Proximal tubules, and Cell Nuclei.

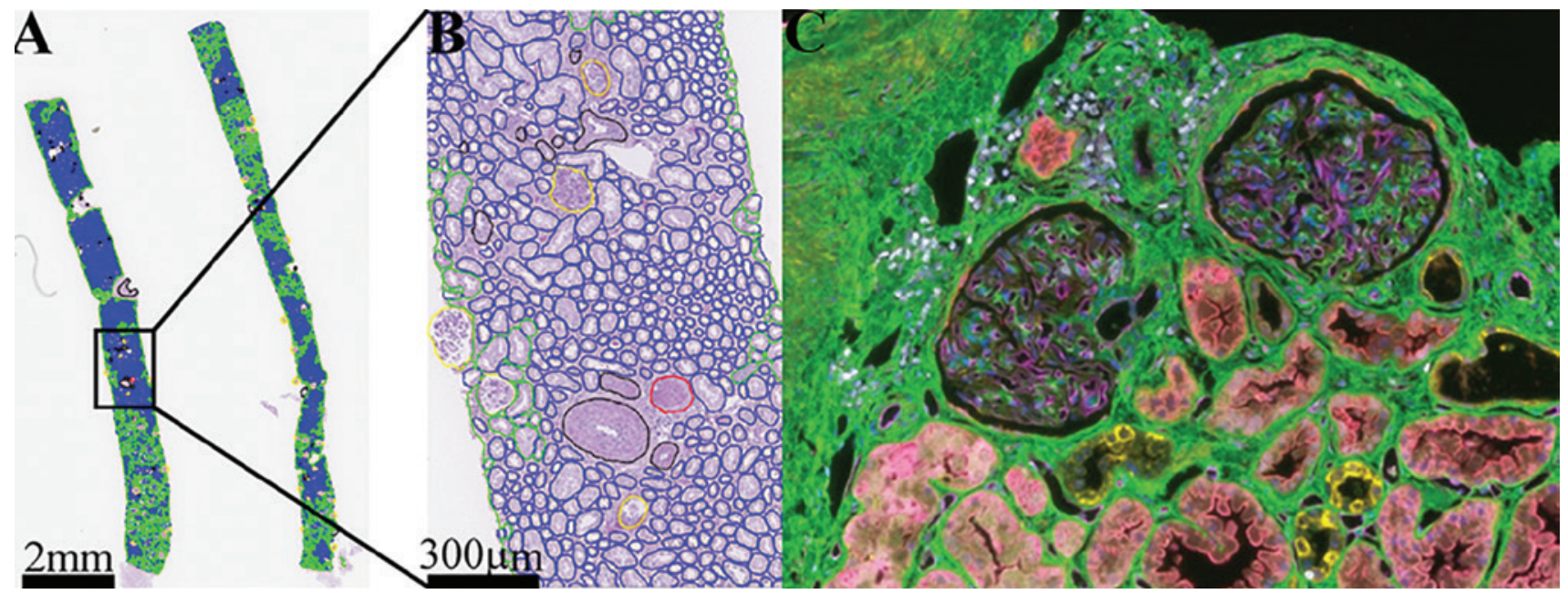

Reprinted courtesy of Brandon Ginley and Kuang-Yu Jen (University at Buffalo, NY, USA and University of California, CA, USA) 
The road to the success of ML tools is not without its obstacles. In CNN, we need to start by addressing image noise, both in and out. Several artifacts will variably occur in WSIs and interfere with the correct CNN training and analysis. In a kidney biopsy, the presence of intratubular casts or red blood cells in glomeruli in our WSIs will significantly affect the identification of glomeruli and tubules. Heavy training with human-generated annotations will not be enough to account for all the possible variations. Hence, we need to achieve intelligent solutions to make our CNNs adapt and be consistent. Supervised training implies a significant number of human-generated annotations. Novel interactive annotation techniques have already been designed to accelerate and simplify this process. ${ }^{14}$ In the future, different research groups will find unique solutions to detect the same objects. Once this happens, our best opportunity is not to focus on who is doing it better, but, alternatively, build ontological-based frameworks to combine these methods so all differently quantified morphometry can contribute a meaningful analysis.

At present, most research in the field is accomplished by computer and biomedical engineers. It is necessary to draw the attention of the final consumers of these products: pathologists and nephrologists must get involved in developing and optimizing these tools. Researchers will then have to design user-orientated software to best empower these professionals in using Al to answer essential questions for their practice.

The biopsy's morphometric analysis is indisputably important, but obtaining the same tissue's core molecular-level data is now within our reach. Spatial transcriptomics is a modern technology that provides transcriptomics measured spatially from the same tissue region of the tissue image. This technique could potentially build comprehensive molecular maps and expose unique pathophysiological mechanisms and new therapeutic targets. A key future challenge is to use Al to merge all these vast data sources to our patient's best benefit.

\section{THE FUTURE IS PROMISING}

The time for a new human and machine cooperation is coming. When Garry Kasparov was beaten at chess in 1997 by the brute calculating force of Deep Blue, many thought the game's potential was exhausted. Just recently, a ML method applied to chess has shaken centuries of theory and certainties to the point that its creative ideas are forcing humans to relearn the game. We believe the same can happen in the field of nephropathology. There is probably much to discover in the subtle and subvisual pathological world that will guide us toward better medical care.

Disclosure of potential conflicts of interest: none declared

\section{References}

1. Esteva A, Kuprel B, Novoa RA, Ko J, Swetter SM, Blau HM, et al. Dermatologist-level classification of skin cancer with deep neural networks. Nature. 2017;542(7639):115-8.

2. Li X, Zhang S, Zhang Q, Wei X, Pan Y, Zhao J, et al. Diagnosis of thyroid cancer using deep convolutional neural network models applied to sonographic images: a retrospective, multicohort, diagnostic study. Lancet Oncol. 2019 Feb;20(2):193-201.

3. Campanella G, Hanna MG, Geneslaw L, Miraflor A, Werneck Krauss Silva V, Busam KJ, et al. Clinical-grade computational pathology using weakly supervised deep learning on whole slide images. Nat Med. 2019 Aug:25(8):1301-9.

4. Savoy M. IDx-DR for Diabetic Retinopathy Screening. Am Fam Physician. 2020 01;101(5):307-8.

5. Evans AJ, Bauer TW, Bui MM, Cornish TC, Duncan H, Glassy EF, et al. US Food and Drug Administration Approval of Whole Slide Imaging for Primary Diagnosis: A Key Milestone Is Reached and New Questions Are Raised. Arch Pathol Lab Med. 2018;142(11):1383-7.

6. Barisoni L, Gimpel C, Kain R, Laurinavicius A, Bueno G, Zeng C, et al. Digital pathology imaging as a novel platform for standardization and globalization of quantitative nephropathology. Clin Kidney J. 2017 Apr 1:10(2):176-87.

7. Hanna MG, Reuter VE, Samboy J, England C, Corsale L, Fine SW, et al. Implementation of Digital Pathology Offers Clinical and Operational Increase in Efficiency and Cost Savings. Arch Pathol Lab Med. 2019;143(12):1545-55.

8. Ginley B, Lutnick B, Jen K-Y, Fogo AB, Jain S, Rosenberg A, et al. Computational Segmentation and Classification of Diabetic Glomerulosclerosis. J Am Soc Nephrol. 2019 Oct 1;30(10):1953-67.

9. Ginley B, Jen K-Y, Rosenberg A, Yen F, Jain S, Fogo A, et al. Neural Network Segmentation of Interstitial Fibrosis, Tubular Atrophy, and Glomerulosclerosis in Renal Biopsies. ArXiv200212868 Cs Eess Q-Bio [Internet]. 2020 Feb 28 [cited 2020 Dec 5]; Available from: http://arxiv.org/ abs/2002.12868

10. Wang CJ, Wetmore JB, Crary GS, Kasiske BL. The Donor Kidney Biopsy and Its Implications in Predicting Graft Outcomes: A Systematic Review: Deceased Donor Kidney Biopsy. Am J Transplant. 2015 Jul;15(7):1903-14

11. Teixeira AC, Ferreira E, Marques MG, Rodrigues L, Santos L, Romãozinho C, et al. Pretransplant Biopsy of Marginal Kidneys: Is It Necessary? Transplant Proc. 2019 Jun;51(5):1585-9.

12. Srivastava A, Palsson R, Kaze AD, Chen ME, Palacios P, Sabbisetti V, et al. The Prognostic Value of Histopathologic Lesions in Native Kidney Biopsy Specimens: Results from the Boston Kidney Biopsy Cohort Study. J Am Soc Nephrol. 2018 Aug;29(8):2213-24.

13. Bajema IM, Wilhelmus $S$, Alpers $C E$, Bruijn JA, Colvin RB, Cook HT, et al. Revision of the International Society of Nephrology/Renal Pathology Society classification for lupus nephritis: clarification of definitions, and modified National Institutes of Health activity and chronicity indices. Kidney Int. 2018:93(4):789-96.

14. Lutnick B, Ginley B, Govind D, McGarry SD, LaViolette PS, Yacoub R, et al. An integrated iterative annotation technique for easing neural network training in medical image analysis. Nat Mach Intell. 2019 Feb;1(2):112-9.

\section{Correspondence to:}

Luís Rodrigues

Nephrology Department, Centro Hospitalar e Universitário de Coimbra, Coimbra, Portugal

E-mail: luis.arodrigues@hotmail.com 